\title{
A Computer-based Decision Support System Used in Identifying Relationship between Oral Symptoms and Systemic Diseases
}

\author{
Dana V. Ghiga ${ }^{1}$, Marius D. Petrişor ${ }^{2}$, Anca Bacârea ${ }^{3}$, Vladimir C. Bacârea ${ }^{1}$, Marius Ş. Măruşteri ${ }^{2}$, \\ Bogdan A. Haifa ${ }^{2}$, Dan Ş. Sîmpălean ${ }^{2}$, Alexandru Şchiopu ${ }^{3}$ \\ 1 Department of Medical Research Methodology, \\ University of Medicine and Pharmacy, \\ 38, Gh. Marinescu Street, Tîrgu Mureş, 540000, Romania, \\ dana_ghiga@yahoo.com \\ 2 Department of Medical Informatics and Biostatistics, \\ University of Medicine and Pharmacy, \\ 38, Gh. Marinescu Street, Tîrgu Mureş, 540000, Romania, \\ 3 Department of Pathophysiology, \\ University of Medicine and Pharmacy, \\ 38, Gh. Marinescu Street, Tîrgu Mureş, 540000, Romania,
}

\begin{abstract}
The aim of this study is to present a dental patient management software application module that offers the possibility to record the oral symptoms of systemic diseases and presents a presumptive diagnosis. We implemented a web application for patients' management in the dental office which includes such a module that besides presenting a presumptive diagnosis also provides detailed information regarding the disease and the recommended laboratory investigations. The implemented software application allows the selection of the oral lesions revealed during intraoral examination and once the oral lesion has been selectedthe application displays the list of the systemic diseases which presents the selected oral lesion as an oral symptom. After displaying the probable systemic diseases, the user can learn more information about a particular disease by clicking on its label. An innovative aspect of the proposed application is the existence of the module that allows the presumptive diagnosis of a systemic disease by selecting the oral lesions found during intraoral examination. Also the software application offers information regarding the selected systemic disease etiology, oral signs and symptoms and the laboratory investigations required for the final diagnosis.
\end{abstract}

Keywords: oral manifestations, disease, computer-assisted diagnosis

\section{Introduction}

The use of computers to help health professionals in their work has been studied since the 1950s [1]. Initially, efforts were focused on developing diagnostic systems [2]. Ledley and Lusted [3] were the first to have addressed this possibility. They described the use of punch cards to indicate the relationship between diseases and their manifestations. An experimental prototype was described in a later publication [4]. Issues related to limitations of scientific basis and reluctance by practitioners to accept a system that was not integrated into regular workflow prevented the establishment of the system on large scale [5].

In the last decadescomputers have been introduced in the medical field, but in recent years scientists are trying to make the use of computers more efficient by developing a variety of applications including image analysis [6], color analysis as an alternative to the spectrophotometer [7],hospital management integrated solutions. The use of a computer has also become mandatory in the dental office.
Nowadays, a computer with Internet access has become an essential tool in the dental office.

In dental practice, in order to improve health care, numerous software applications have been developed with the most varied uses, from "simple" data storage to processing and analysis or three-dimensional reconstruction and simulation. Nowadays, for almost every clinical and technological phase, there is a computerized system which serves the physicians or the technicians in their work.

Currently available systems do not offer any possibility of recording the oral lesions that may be present in the oral cavity, due to systemic disease.

Many problems in dental practice are not trivial or simple, and therefore require rigorous research efforts. Without well-founded methodological approaches, computer technology will not achieve the expected performances, those of helping the physicians and patients.

Integrated computer systems, must be designed around the work of medical staff and should 
allow them to better focus on patient care. Such systems must provide information when and where necessary, minimize duplication of data entry and their indirect and should be easy to use.

The aim of this study is to present a module from a patient management software application in the dental office that offers the possibility of recording the oral symptoms of systemic diseases and presenting a presumptive diagnosis.

\section{Material and Method}

We implemented a web application for patients' management in the dental office. It uses Apache for the web server and MySQL as a database management system.

The coding was made in PHP 5 and an open source object oriented Javascript framework named Qooxdoo. Using this framework, we created the graphical user interface (GUI). After the compilation, the framework created a single HTML file (index.html) and a JS file which is accessed on page load. The JS file contains all the functions necessary for the browser to "draw" the GUI. One of the advantages of using Qooxdoo is that the GUI looks and performs exactly the same on every major browser and operating system.

Communication with the database is achieved using a PHP backend, which executes all the queries (synchronous and asynchronous), sent from the GUI. The received information is displayed without the need of accessing a different URL, the application running on the page.
The hosting is on a Linux system (Debian 5). The application can be accessed through any web browser with Javascript turned on, capable of handling AJAX requests. These kind of asynchronous queries are used for populating lists and validating data input.

\section{Data Storage}

All the patients' data are stored into a database consisting of 9 tables (41 fields). We found MySQL to be suitable for this kind of application, given the need for a relational database and a fast response time [8].

We present the software application module that enables recording the oral symptoms of systemic diseases and presents a presumptive diagnosis. This module is part of an integrated solution for patients' management in the dental office. After the user selects the oral lesions found during the oral exam, the PHP backend executes the queries needed to associate this data with a systemic disease (Figure 1).

\section{Results}

The Graphical User Interface consists of 9 tabs.

In Personal Data tab, the user fills in patient's personal information (first and last name, personal identification number, birth date and sex). Antecedents tab is divided in two categories: General Antecedents (physiological and pathological) and Local Antecedents (dental caries, dental extractions, signs of periodontitis, previous dental treatment,

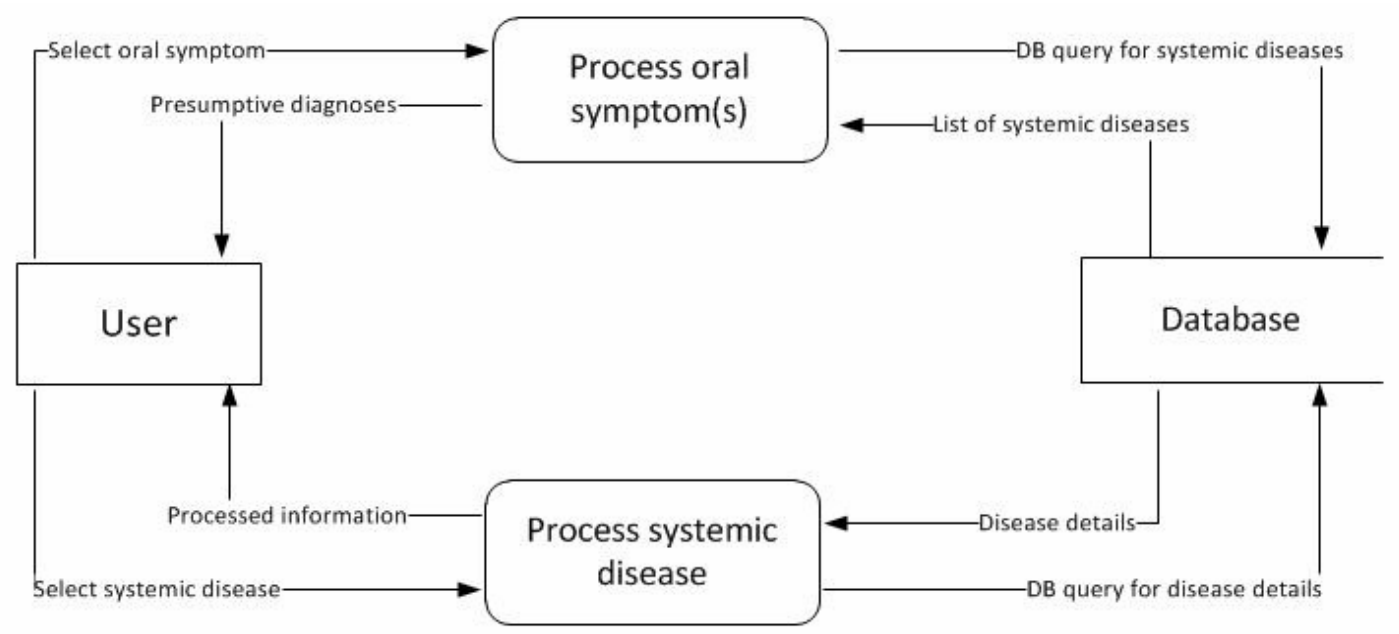

Figure 1. Data flow diagram for oral symptoms and systemic diseases module 
parafunctional occlusion and vicious habits).

History tab contains patient complaints concerning current visit, disease history, family medical history (general and dento-maxillary).

In Local medical examination tab there will be registered each tooth health status, including missing teeth and any periodontal lesions.

Paraclinical section offers the possibility of x-rays and other images storage for future references.
Oral lesions section is divided into two panes, the first contains the list of oral lesions that can be seen in the oral cavity and in the second one will appear a list with the probable systemic diseases. After selecting one oral lesion, the second pane displays the list of systemic diseases which presents the selected oral lesion as an oral symptom (Figure 2).

Selecting more oral lesions reduces the number of list items (Figure 3).

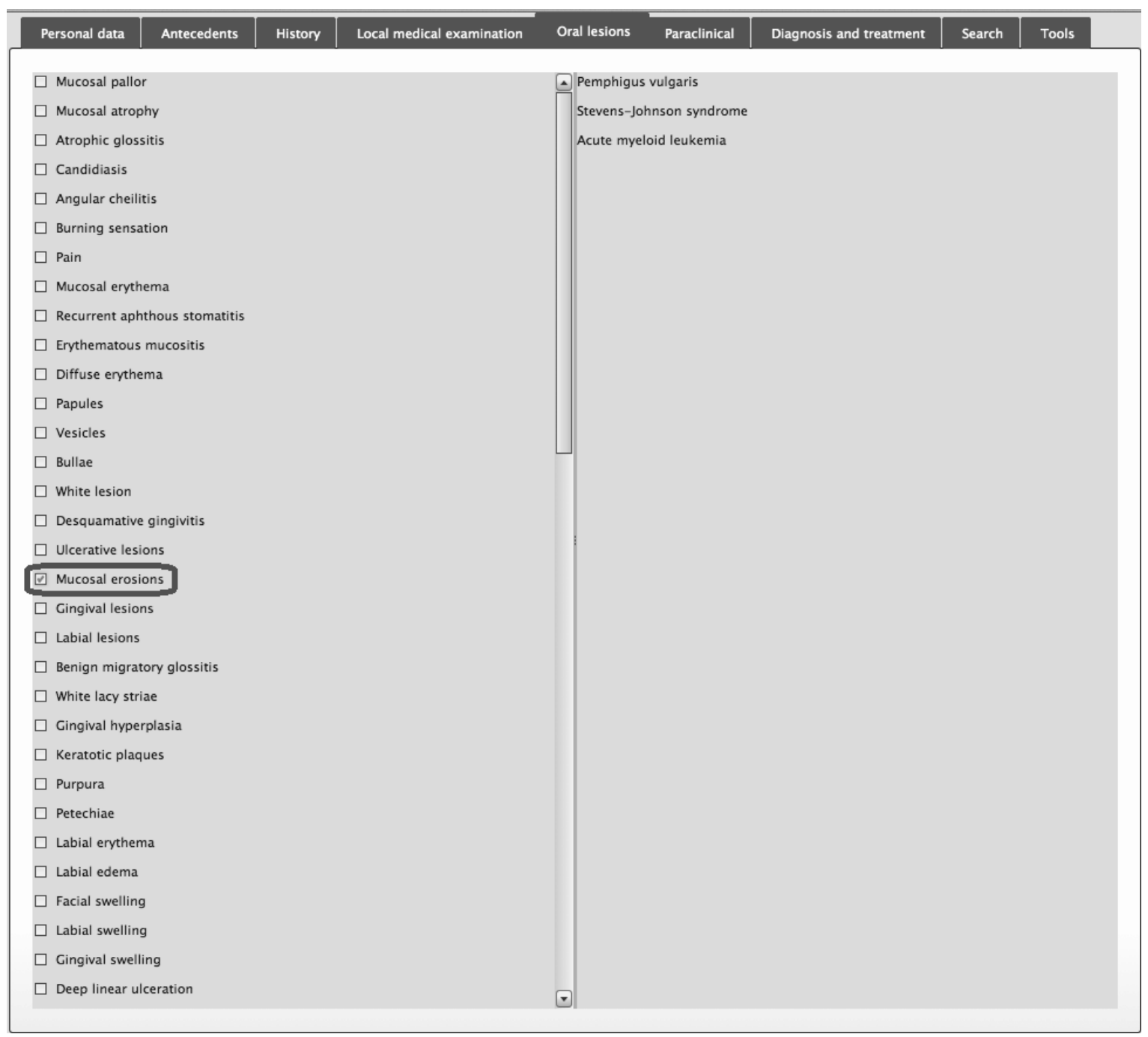

Figure 2. Possible systemic diseases with the one oral lesion selected

The user is able to record the diagnosis and the treatment for each visit. This is achieved in Diagnosis and treatment tab.

After filling in the fields on the previous tabs (personal data, history, local history and examination), the presented software application allows the selection of the oral lesions revealed during intraoral examination.
After displaying the probable systemic diseases, the user can learn more information about a particular disease by clicking on its label. By carrying out this operation, a new window containing the etiology, the oral signs and the oral symptoms of the selected systemic disease and the laboratory investigations that are recommended for the diagnosis of the systemic disease suspected, will be displayed (Figure 4). 
The developed software application contains a "Tools" section. This section allows the dentist to input into the database new oral lesions and systemic diseases and to create new associations between oral lesions and systemic diseases. Initially the dentist seeks out the systemic disease and the oral lesions in the database and if those are not found they can be return a set of records that corresponds to the search criteria.

\section{Discussion}

It is a common misconception among both dental professionals and medical doctors that

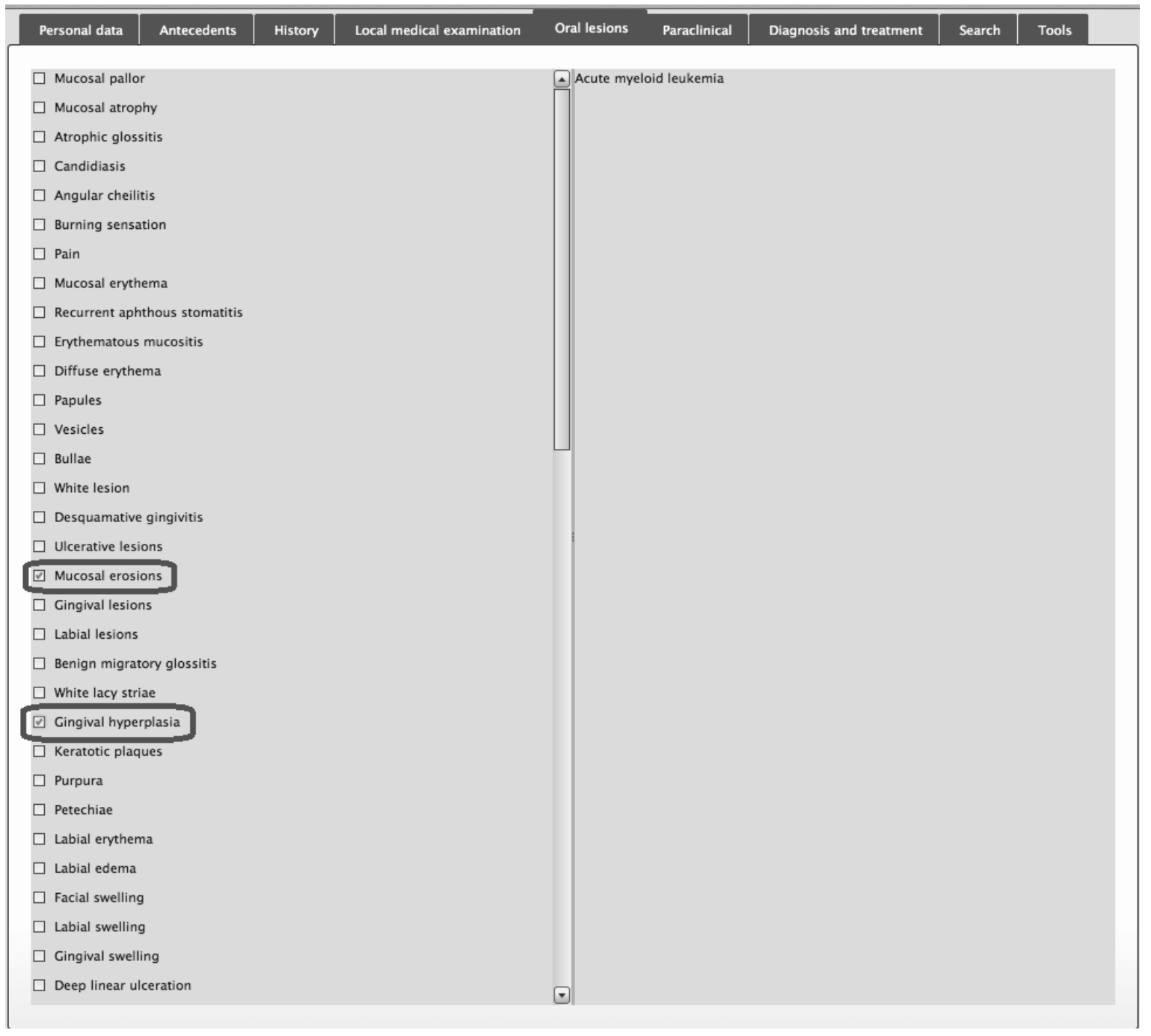

Figure 3. Possible systemic diseases with multiple oral lesions selected

entered manually by typing in the systemic disease field and then clicking the "Add” button. The same operation is performed for introducing a new oral lesion.In order to create a new association the user will select the oral lesion and the systemic disease and hit the „Create” button.

Also the application presents a Search tab. Using this feature, the user has the possibility to search a patient by its name. The search will oral diseases refer only to dental caries and periodontal disease [9].

Many systemic diseases present oral symptoms. Those can be the first or the only sign of a systemic disease or an underlying disease process presence. It is therefore important that an appropriate oral examination should be an integral part of patient assessment in a dental office and that the dentist should know and recognize these lesions leading this way to an early diagnosis of certain systemic diseases [10]. 
While the best systems are far from fully realized, the technology has undoubtedly made some progress.

White [11] is one of the pioneers of computer based decision support system, contributing to their success in dentistry. In his research, White studied more than 30 decision support systems, identifying 7 dentistry subdomains where these systems were used at that time. These subdomains are Dental emergencies and trauma, Oro-facial pain, Oral medicine, Oral radiology, Orthodontics, Pulpal diagnosis, Pulpal diagnosis.

Regarding dental software applications, dentists need various information in making a diagnosis and treatment decisions, andthe increasing prevalence of chronic diseases lead to challenges in understanding of oral-systemic interactions [12].

Nowadays, a wide variety of commercial dental practice management software is available. However some authors [13] revealed significant usability problems especially for novice users. Usability problems can result in negative consequences in the way practitioners make clinical decisions [14], how efficiently practitioners interact with information about patients [15] and how dental professionals and medical doctors collaborate [16].

The user interacts with the system by accessing its interface and as others studies showed [17] the acceptance level of an application is higher if the interface is easy to use and learn.

Considering the usability problems in other solutions, we developed an easy to use

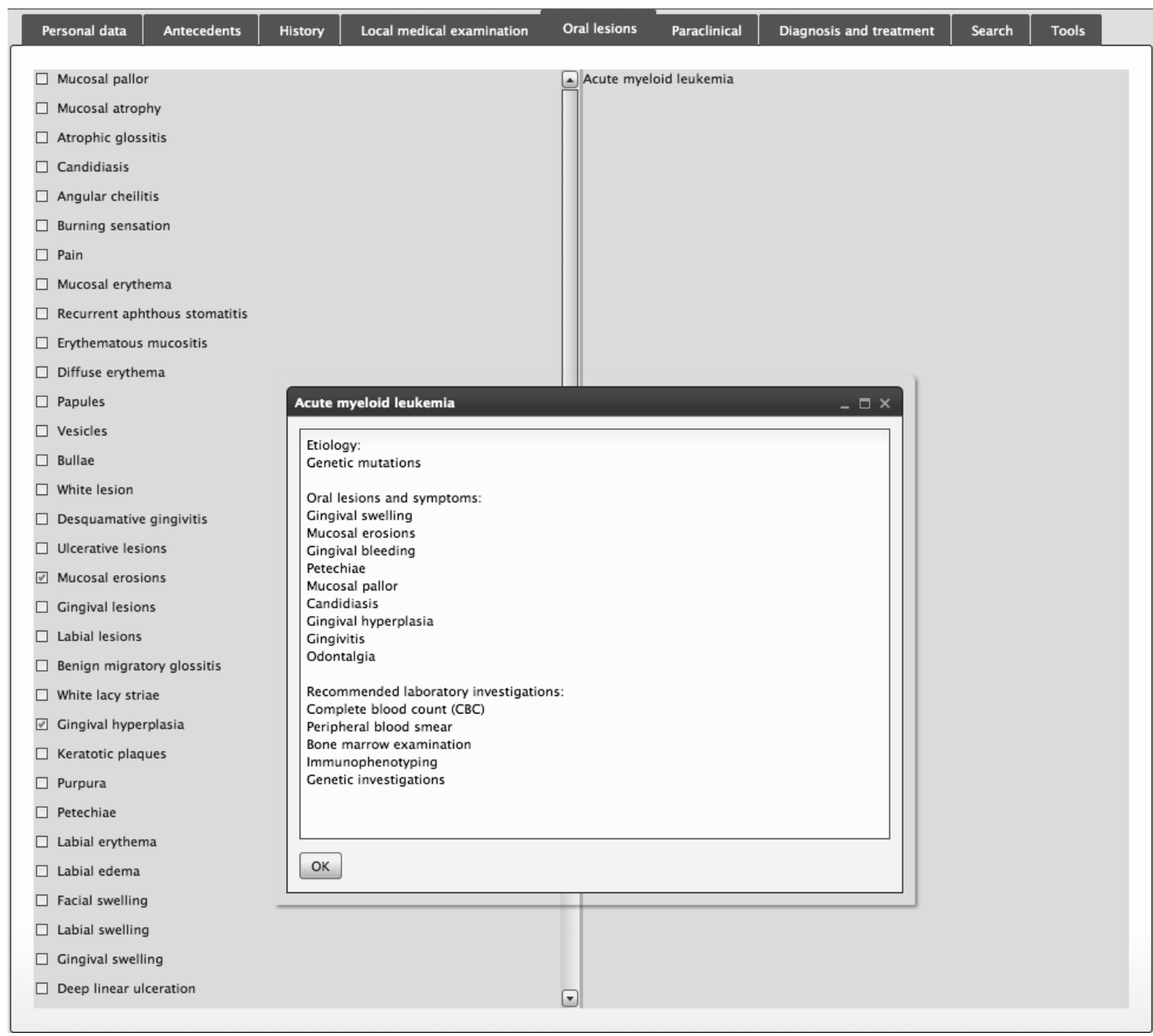

Figure 4. The complete symptoms list of the possible systemic disease and the recommended laboratory investigations 
application that does not require any previous computer use trainings.

Most of the existing software solutions focus on maintaining an accurate patient records database. These applications allow keeping track of patients' previous visits, interventions, treatments, payments and also offer the possibility to make further appointments.

None of the computer applications we encountered so far contained a decision support system regarding systemic diseases presumptive diagnostic based on the oral signs and symptoms.

Given the importance of noticing oral signs of systemic diseases, we considered very important to integrate in patient management software a module that enables this feature.

To develop such an application we chose to use a web platform due to the multiple advantages a web application may have over a desktop one. First of all every major operating system has at least one web browser capable of accessing the application and no changes are made on the client computer during the use of the application. Also the client computer resources are used minimally, data is centralized, secured and easy to backup and the web application is easier to update and maintain.

Being a web application, the described solution can be accessed simultaneously by more than one user.

The application is built in such a way that can be easily expended by integrating newly written modules, such as accountancy and further patients' appointments.

The only disadvantages we encountered concerning a web application refers to the need of a powerful server capable of handling the amount of data transferred and a stable and fast internet connection. This issue can be addressed by separating the services on multiple servers, for example a server for the database and one for the web service.

Similar to existing dental informatics systems, the proposed software application allows the recording of patients' personal data, personal and family history, case history and oral examination, paraclinical investigations each in separate screens. However we didn't focus on patients' payments and accountancy.
The application offers the possibility new oral signs and symptoms and systemic diseases to be entered into the database.

During the selection of oral lesions, the application returns a presumptive diagnosis of the systemic disease. For each systemic disease, the software application offers information regarding etiology, oral signs and symptoms and the laboratory investigations that are recommended for the final diagnosis.

Many problems in dental practice are not trivial or simple, and therefore require rigorous research efforts. Without robust methodological approaches, computer technology will not achieve the expected performances, namely to assist physicians and patients.

Vikram et al [18] concluded that in order to ensure a long term use of the computer based decision support system, the developer must take into consideration users' feedback.

Even though we think that the proposed decision support system would be useful in dental office, we have to take into consideration users' receptivity to such systems.

As other authors stated [18], we agree that an important component of the computer based decision support system integration in dentistry is represented by the clinical experience and knowledge of the dentist who uses such systems.

\section{Conclusions}

An important aspect of patient's dental examination is the identification by the dentist of the oral lesions that are signs of a systemic disease.

An innovative aspect of the proposed application is the existence of a module that allows the presumptive diagnosis of a systemic disease by selecting the oral lesions found during intraoral examination.

Also the software application offers information regarding the selected systemic disease etiology, oral signs and symptoms and the laboratory investigations required for the final diagnosis. 


\section{Acknowledgement}

This paper is partly supported by the Sectorial Operational Programme Human Resources Development (SOP HRD), financed from the European Social Fund and by the Romanian Government under the contract number POSDRU 80641.

\section{REFERENCES}

1. MENDONÇA, E. A., Clinical Decis ion Support $S$ ystems: Perspectives in Dentistry. Journal of Dental Education, vol. 68(6), 2004, pp. 589-597.

2. MILLER. R. A., Medical Di agnostic Decision Support Sys tems - Past, Present, and Future: A Threade d Bibliography an d Brie f Com mentary. Journal of American Medical Informatics Association, vol. 1(1), 1994, pp. 8-27.

3. LEDLEY, R. S., L. B. LUSTED, Reasoning Found ations of Me dical Diagnosis, Science, vol. 130, 1959, pp. 9-21.

4. WARNER, H. R., A. F. TORONTO, L. VEASY, Experience with Baye 's Theorem for Compute $r$ Diagnosis of Congenital Heart Disease. Annals of the New York Academy of Science, vol. 115, 1964, pp. 2-16.

5. MUSEN, M. A., Y. SHAHAR, E. H. SHORTLIFFE, Clinical Decisi onSupport S ystems, in Shortliffe E. H., Perreault L. E, Wiederhold G, Fagan L. $M$, eds. Medical informatics: computer applications in health care and biomedicine. 2nd ed. New York, SpringerVerlag, 2001, pp. 573-609.

6. CHIOREAN, L., L. SUTA, M. VAIDA, M. HEDESIU, Medical Fusion

Components for a Web Dedic ated Application, SIC, vol. 19(4), 2010, pp. 435-444.

7. HAIFA, B., V. BACÂREA, O. IACOB, T. CĂLINICI, A. ŞCHIOPU, Com parison between Digital Image Processing and Spectrophotometric Measurements Methods. Applic ation in Electrophoresis Inter pretation, Applied
Medical Informatics, vol. 28(1), 2011, pp. 29-36.

8. MUJI, M., R. V. CIUPA, D. DOBRU, C. BICA, P. OLAH, V. BACAREA, M. MARUSTERI, Database Design Patterns for He althcare Inform ation Syste ms, International Conference on Advancements of Medicine and Health Care Through Technology, vol. 26, 2009, pp. 63-66.

9. U.S. Department of Health and Human Services. Oral Health in America: A Report of the Surgeon Gene ral. Rockville, MD: U.S. Department of Health and Human Services, National Institute of Dental and Craniofacial Research, National Institutes of Health, 2000.

10. ISLAM, N. M., I. BHATTACHARYYA, D. M. COHEN, Common Oral Manifestations of Systemic Disease Otolaryngologic Clinics of North America Oral Medicine, vol. 44(1), 2011, pp. 161-182.

11. WHITE, S. C., Decision-support systems in dentistry. Journal of Dental Education, vol. 60(1), 1996, pp. 47-63.

12. SONG, M., H. SPALLEK, D. POLK, T. SCHLEYER, T. WALI, How Information Sys tems should Support the Information Needs of Gene ral Dentists in Cli nical Set tings: Suggestions from a $Q$ ualitative Study. BMC Medical Informatics and Decision Making, vol. 10, 2010, pp. 7-16.

13. THYVALIKAKATH, T. P., V. MONACO, H. B. THAMBUGANIPALLE, T. SCHLEYER, A Usabi lity $E$ valuation of Four Commercial Dental Computer-based Patient Rec ord Systems . JADA, vol. 139(12), 2008, pp. 1632-1642.

14. ELTING, L. S., C. G. MARTIN, S. B. CANTOR, E. B. RUBENSTEIN, Influence of Data D isplay Formats on Physician I nvestigators' Decisi ons to Stop $C l$ inical $T r$ ials: $P$ rospective Trial with Repe ated Me asures. BMJ, vol. 318(7197), 1999, pp. 1527-1531. 
15. NYGREN, E., J. C. WYATT, P. WRIGHT, Helping Clinicians to Find Data and Avoid Delays . Lancet, vol. 352(9138), 1998, pp. 1462-1466.

16. PRATT, W., M. C. REDDY, D. W. MCDONALD, P. TARCZY-HORNOCH, J. H. GENNARI, Incorporating I deas from Computer-supported Cooperative Work. Journal of Biomedical Informatics, vol. 37(2), 2004, pp. 128-137.
17. SUDUC, A.-M., M. BÎZOI, L. DUȚĂ, G. GORGHIU, Interface Architecture for a Web-Based Group D ecision Support System, SIC, vol. 18(3), 2009, pp. 241-246.

18. VIKRAM, K., F. R. KARJODKAR, Decision Support Sys tems in D ental Decision Making: A n Introduc tion, Journal of Evidence Based Dental Practice, vol. 9(2), 2009, pp. 73-76. 